\title{
Anesthetic management of a parturient with active tuberculous meningitis
}

\author{
Suman Rajagopalan*, Raja R Palvadi and C. LaToya Mason \\ Department of Anesthesiology, Baylor College of Medicine, Houston, TX, USA
}

\begin{abstract}
Tuberculous meningitis (TBM) results from the infection of the meninges and the cerebrospinal fluid with Mycobacterium tuberculosis. The diagnosis and treatment of TBM is challenging due to its protean manifestations. We present the anesthetic management of a parturient with advanced TBM in whom urgent cesarean delivery was performed due to a non-reassuring fetal status. The patient's disease course was remarkable for altered mental status, seizures, isoniazid-induced hepatitis and hydrocephalus requiring ventriculo-peritoneal shunt placement. General endotracheal anesthesia was successfully performed that resulted in the safe delivery of a live infant.
\end{abstract}

\section{Introduction}

Tuberculosis (TB) of the central nervous system is a severe form of extra-pulmonary $\mathrm{TB}$ and encompasses three clinical categories: tuberculous meningitis, intracranial tuberculoma, and spinal tuberculous arachnoiditis. Tuberculous meningitis (TBM) results from the infection of the meninges and the cerebrospinal fluid (CSF) with the acid-fast bacillus Mycobacterium tuberculosis. According to the Centers for Disease Control and Prevention, TBM accounts for about 1 percent of all cases of TB and 6 percent of all extra-pulmonary TB infections in immuno-competent individuals [1]. Whereas the typical manifestations of TBM include an insidious-onset subacute febrile illness that progresses to meningismus, and worsening of the mental status, atypical presentations that mimic acute pyogenic meningitis or a neurodegenerative disorder with progressive dementia have also been described [2-5]. Pregnant women have been reported to have an increase in the risk for the extra-pulmonary forms but not the pulmonary form of TB [6]. The anesthetic management of a parturient with TBM can be challenging due to the increased risk of elevated intracranial pressure (ICP), seizures, and aspiration. In this report, we discuss the successful anesthetic management in a female who required an emergency cesarean section.

\section{Case description}

A 26-year-old Gravida 2, Para 1 woman in her twenty eighth week of gestation presented with a history of altered mental status and seizures. A diagnosis of TBM was established based on the cerebrospinal fluid (CSF) examination that showed mononuclear pleocytosis, elevated protein, low glucose, and a polymerase chain reaction that revealed Mycobacterium tuberculosis nucleic acid. A Directly Observed Therapy (DOT) regimen consisting of isoniazid, rifampicin, pyrazinamide, and ethambutol was initiated. Within two weeks of the start of the therapy, she developed isoniazid-induced hepatotoxicity and this drug was discontinued. In spite of therapy with the remaining three medications, there was no improvement in her altered mental status and she continued to be withdrawn and confused. The magnetic resonance imaging of the brain revealed hydrocephalus (Figure 1A). A ventriculo- peritoneal (VP) shunt was placed to relieve the increased ICP (Figure $1 \mathrm{~B})$. At 35 weeks of gestation, she was diagnosed to have nonreassuring fetal status on routine fetal surveillance and was brought to the operating room for an emergent cesarean delivery. The decision to perform the delivery under general anesthesia was made based on the emergent nature of the delivery, mental status changes of the patient and active CNS infection. Aspiration prophylaxis with nonparticulate antacid was given and rapid sequence induction of general anesthesia was achieved using propofol. Succinylcholine was used to facilitate intubation despite her elevated ICP, as the patient was not adequately nil orally. Intubation was achieved with the use of a Macintosh 3 blade. Anesthesia was maintained with sevoflurane in $100 \%$ oxygen. Fentanyl was titrated after the delivery of the baby to avoid narcotization of the new born. A live infant was delivered with Apgar score of 3 at 1 minute
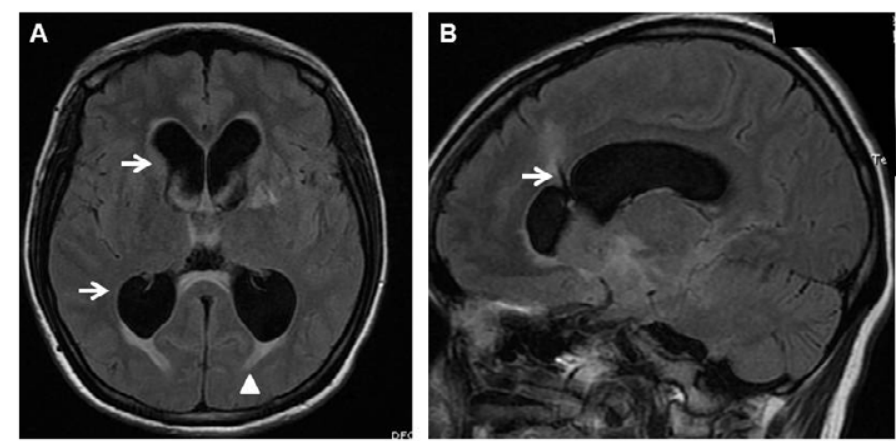

Figure 1. A. Magnetic resonance imaging showing hydrocephalus with dilatation of the frontal and occipital horns of the lateral ventricles (arrows) and the subependymal extravasation of CSF (arrowhead). B. VP shunt was placed to relieve the intracranial pressure (arrow).

Correspondence to: Suman Rajagopalan, Department of Anesthesiology, Baylor College of Medicine, 1709 Dryden, Suite 1700, Houston, Texas 77030, USA, Tel: +1-713-873-3430; E-mail: srajagop@bcm.edu

Key words: Tuberculous meningitis, parturient, cesarean delivery

Received: March 03, 2015; Accepted: March 20, 2015; Published: March 22, 2015 
and 7 at 5 minutes. The patient had no intra-operative complications and was extubated awake at the completion of the procedure. The patient was able to maintain her airway and was transferred to the floor for further care. She was subsequently discharged home on 4 anti-TB drug regimen. Five months after the delivery, the patient expired due to complications of TBM $[7,8]$.

\section{Discussion}

TBM, the most dominant form of central nervous system TB results from the hematogenous spread of Mycobacterium tuberculosis from a primary focus or a late reactivation of infection from elsewhere in the body. The seeding of the bacilli to the meninges or brain parenchyma results in the formation of small subpial or subependymal foci of caseous lesions termed 'Rich's foci' [9]. Rupture of these lesions into the subaracnoid space results in the infection of the meninges and leads to an intensive hypersensitivity reaction and inflammation that is most marked at the base of the brain [9-10].This leads to proliferative arachnoiditis that can result in cranial neuropathies, vasculitis that can cause strokes, and impedance to the flow of CSF that can lead to hydrocephalus [10].

The typical manifestations in TBM can be categorized into three phases: 1) Prodromal phase with malaise, headaches and a low-grade fever, 2) meningitis phase with signs of meningeal irritation, altered mental status, and entrapment cranial neuropathies, and 3) paralytic phase with progressive worsening that includes stupor, coma, seizures, and motor deficits that are a result of stroke $[3,4,11]$. The diagnosis of TBM is established by the examination of CSF that typically shows lymphocytic pleocytosis, increased protein, and decreased glucose concentrations. The presence of Mycobacterium tuberculosis in CSF can be accomplished by smear and culture for acid-fast bacilli, or nucleic acid amplification tests like polymerase chain reaction that detect Mycobacterial DNA.

In pregnant women, the course of TB in general is more indolent with the main symptoms being fatigue, malaise and weight loss [6]. The diagnosis of TBM especially in the early stages, can hence be delayed due to the non-specific manifestations in the prodromal phase [12]. A high-index of clinical suspicion is required to make the diagnosis. The initiation of treatment in the early stages of the disease is critical in reducing mortality and morbidity and empiric therapy should be promptly started in those with a suspicion of TBM even prior to confirmation of diagnosis [13]. The treatment of TBM in pregnancy consists of a two-month period of intensive therapy with four drugs, isoniazid, rifampicin, pyrazinamide, and ethambutol, followed by a prolonged continuation phase lasting seven to 10 months with rifampicin and isoniazid $[14,15]$. Although the effect of pyrazinamide on the fetus is not fully known, the World Health Organization (WHO) recommends its administration as part of standard regimen for treating TB in pregnant women [16]. Isoniazid, rifampicin and ethambutol are considered 'acceptable for use' in pregnancy although the risk of isoniazid -induced hepatitis is increased in pregnancy and the early postpartum period [17].

\section{Anesthetic considerations in parturient with TBM}

The presence of an active infection and complications including increased ICP, seizures, and strokes are the factors that decide the choice of the anesthetic technique. Whereas regional anesthesia is the preferred technique for delivery by cesarean section, the presence of an active infection in the meninges and increased ICP preclude its usage in parturients with TBM [18]. The risk of neuraxial puncture in patients with increased ICP can result in tentorial herniation [19]. Although it can be argued that epidural anesthesia could be provided as it does not penetrate the meninges, the risk of accidental dural puncture is always a possibility. Injection of large volumes of a local anesthetic into the epidural space in a patient with increased ICP can further increase the ICP [20]. The presence of neurological deficits is also a relative contraindication for neuraxial techniques.

In obstetric patients with VP shunts, both regional and general anesthetic techniques have been successfully performed. The concerns of performing neuraxial techniques in those with a VP shunt are related to the functioning of the shunt (baseline and after performance of neuraxial technique), changes in ICP, risk of meningitis, herniation, and post-dural puncture headache $[21,22]$. Our patient presented with a VP shunt in situ, an active meningeal infection, and altered mental status. Hence, a decision to proceed with general anesthesia with endotracheal intubation was made.

General anesthesia can increase ICP during laryngoscopy, intubation, and emergence from anesthesia. In addition, there is the added risk of difficult and/or failed intubation and pulmonary aspiration. Premedication with an $\mathrm{H}_{2}$-receptor antagonist and oral administration of non-particulate antacid must be considered in pregnant patients. To minimize the risk of hypoxemia during induction, pre-oxygenation with a tight fitting mask is essential. The induction can be safely performed with either thiopentone or propofol. Narcotics used with induction agents are known to both decrease the sympathetic response of intubation and prevent the increase in ICP. However, narcotics are avoided until the delivery of the infant to prevent respiratory depression in the neonate. Rapid sequence intubation with application of cricoid pressure is a standard component for the induction of all pregnant patients [23]. In the presence of increased ICP or neurological deficits, succinylcholine may be avoided and rocuronium may be used as an alternative. However, succinylcholine still remains the best agent for achieving rapid neuromuscular blockade in patients with an anticipated difficult airway. Hyperventilation to decrease the ICP is avoided in pregnant patients as it can decrease the uterine blood flow [24]. Volatile agents are generally used for the maintenance of anesthesia. Using concentrations of volatile agents greater than one minimum alveolar concentration may result in marked uterine relaxation, bleeding, and elevations of the ICP. Excessive coughing and gagging during the emergence from anesthesia should be avoided to prevent further increases in the ICP.

In summary, TBM results in a high morbidity and mortality. A high-index of suspicion is needed for the diagnosis in a pregnant patient. Early diagnosis and treatment are of paramount importance. General endotracheal anesthesia is generally safe and is the preferred anesthetic technique in the management of such patients with active tuberculous meningitis.

\section{References}

1. CDC, reported tuberculosis in the United States, 2004. Atlanta, GA. US Department of Health and Human Services, CDC. September 2005.

2. Hinman AR (1967) Tuberculous meningitis at Cleveland Metropolitan Genera Hospital 1959 to 1963. Am Rev Respir Dis 95: 670-673. [Crossref]

3. Kennedy DH, Fallon RJ (1979) Tuberculous meningitis. JAMA 241: 264-268 [Crossref]

4. Kent SJ, Crowe SM, Yung A, Lucas CR, Mijch AM (1993) Tuberculous meningitis: a 30-year review. Clin Infect Dis 17: 987-994. [Crossref]

5. Udani PM, Dastur DK (1970) Tuberculous encephalopathy with and without meningitis Clinical features and pathological correlations. J Neurol Sci 10: 541-561. [Crossref] 
6. Llewelyn M, Cropley I, Wilkinson RJ, Davidson RN (2000) Tuberculosis diagnosed during pregnancy: a prospective study from London. Thorax 55: 129-132. [Crossref]

7. Baidya DK, Trikha A, Menon S, Garg R (2011) Anaesthetic management of emergency caesarean section in a patient with seizures and likely raised intracranial pressure due to tuberculous meningitis. Anaesth Intensive Care 39: 951-953. [Crossref]

8. Prévost MR, Fung Kee Fung KM (1999) Tuberculous meningitis in pregnancy-implications for mother and fetus: case report and literature review. J Matern Fetal Med 8: 289-294. [Crossref]

9. Rich AR, McCordock HA (1933) Pathogenesis of tuberculous meningitis. Bull Johns Hopkins Hosp. 52: 2-37.

10. Dastur DK, Manghani DK, Udani PM (1995) Pathology and pathogenetic mechanisms in neurotuberculosis. Radiol Clin North Am 33: 733-752. [Crossref]

11. Farinha NJ, Razali KA, Holzel H, Morgan G, Novelli VM (2000) Tuberculosis of the central nervous system in children: a 20-year survey. J Infect 41: 61-68. [Crossref]

12. Doveren RF, Block R (1998) Tuberculosis and pregnancy--a provincial study (19901996). Neth J Med 52: 100-106. [Crossref]

13. Hsu PC, Yang CC, Ye JJ, Huang PY, Chiang PC, et al. (2010) Prognostic factors of tuberculous meningitis in adults: a 6 -year retrospective study at a tertiary hospital in northern Taiwan. J Microbiol Immunol Infect 43: 111-118. [Crossref]

14. (1998) Chemotherapy and management of tuberculosis in the United Kingdom recommendations 1998. Joint Tuberculosis Committee of the British Thoracic Society. Thorax 53: 536-548. [Crossref]

15. Blumberg HM, Burman WJ, Chaisson RE, Daley CL, Etkind SC, et al. (2003) American Thoracic Society/Centers for Disease Control and Prevention/Infectious Diseases Society of America: treatment of tuberculosis. Am J Respir Crit Care Med 167: 603-662. [Crossref]
16. Treatment of tuberculosis: Guidelines for national programmes, 3rd ed. WHO/ CDS/ TB/ 2003.313., in World Health Organization. 2003: Geneva, Switzerland.

17. Franks AL, Binkin NJ, Snider DE Jr, Rokaw WM, Becker S (1989) Isoniazid hepatitis among pregnant and postpartum Hispanic patients. Public Health Rep 104: 151-155. [Crossref]

18. Breitenbach V, Wilson DH (2005) Anesthesia in pregnant patient with intracranial hypertension due to tuberculous meningitis: case report. Rev Bras Anestesiol 55: 90-94. [Crossref]

19. Su TM, Lan CM, Yang LC, Lee TC, Wang KW, et al. (2002) Brain tumor presenting with fatal herniation following delivery under epidural anesthesia. Anesthesiology 96 : 508-509. [Crossref]

20. Grocott HP, Mutch WA (1996) Epidural anesthesia and acutely increased intracrania pressure. Lumbar epidural space hydrodynamics in a porcine model. Anesthesiology 85: 1086-1091. [Crossref]

21. Littleford JA, Brockhurst NJ, Bernstein EP, Georgoussis SE (1999) Obstetrical anesthesia for a parturient with a ventriculoperitoneal shunt and third ventriculostomy. Can J Anaesth 46: 1057-1063. [Crossref]

22. Yu JN (1994) Pregnancy and extracranial shunts: case report and review of the literature. J Fam Pract 38: 622-626. [Crossref]

23. Morris J, Cook TM (2001) Rapid sequence induction: a national survey of practice. Anaesthesia 56: 1090-1097. [Crossref]

24. Cook PT (1984) The influence on foetal outcome of maternal carbon dioxide tension at caesarean section under general anaesthesia. Anaesth Intensive Care 12: 296-302. [Crossref]

Copyright: (C2015 Rajagopalan S. This is an open-access article distributed under the terms of the Creative Commons Attribution License, which permits unrestricted use, distribution, and reproduction in any medium, provided the original author and source are credited. 